\title{
Fusion of Multispectral and Panchromatic Images via Local Geometrical Similarity
}

\author{
Hong LI, Fenxia WU, Xiaobo ZHANG
}

\begin{abstract}
A pansharpening method based on local geometrical similarity is proposed in this paper. According to the observation model, the relationships among low spatial resolution multispectral (LRMS), panchromatic (Pan) and high spatial resolution multispectral (HRMS) images are formulated. In this paper, in order to reduce the color distortion and enhance the spatial information of fused images, we propose a Pan-Sharpening method via Local Geometrical Similarity (PLGS). First, the structure similarity prior within a local region in the Pan image is employed to regularize the solution space to obtain a more accurate solution. Then, the prior is embedded into the LRMS image to enhance the spatial resolution. In order to capture better geometrical structure information, such as orientation information and geometric textures, the steerable kernel is used to calculate the similarity coefficients in a local window. Some experiments are considered on different datasets and the results show that the proposed method can improve the visual effect and the quantitative values.
\end{abstract}

Keywords: local similarity; pansharpening; remote sensing images; steerable kernel

\section{INTRODUCTION}

There are some physical limitations for imaging sensors in remote sensing satellites. Therefore, it is difficult to simultaneously obtain high spatial and spectral resolution images. Besides, due to the need of some remote sensing applications [1], such as object recognition [2], the demand for high spatial resolution multispectral (HRMS) images is steadily growing. Thus, the fusion of panchromatic (Pan) and low spatial resolution multispectral (LRMS) images is becoming more and more important [3].

For the past several decades, a lot of effective and classic fusion algorithms are proposed to enhance the spatial resolution of LRMS images through the information in Pan image. These methods are mainly divided into four categories: component substitution-, arithmetic-, multiscale analysis- and restoration- based methods.

The first category is the Component Substitution method. The intensity-hue-saturation (IHS) transform [4] and the principal component analysis (PCA) transform [5-7] are the methods based on Component Substitution, which transform the interpolated LRMS into a new space obtaining the spatial component that will be replaced by the histogram, matched Pan image. These methods are widely used for pansharpening, because they are easy to implement and fast. Experimental results show that these methods can effectively engender spectral distortion. Thus, some works are proposed to decrease the spectral distortions [8]. The second category is the arithmetic based methods, which combine addition, subtraction, multiplication, and division operations together to enhance the resolution of LRMS images, such as Brovey and $\mathrm{P}+\mathrm{XS}$. The third category is based on Multiscale Resolution Analysis (MRA) and ARSIS concept, which assumes that the lost high frequencies in LRMS images can be obtained from Pan image. Therefore, in these methods, the high frequencies are extracted by some MRA tools, such as decimated wavelet transform (DWT) [9], Laplacian pyramid (LP) [10] and contourlet [11]. According to this idea, the additive wavelet luminance proportional (AWLP) [12] is proposed to use a general fusion formulation and defined injection coefficients, which obtains better performance in spectral preservation. Experimental results show that methods based MRA can effectively enhance the spectral resolution, but slightly reduce the spatial quality of the fused images.

Recently, some methods based on Compressed Sensing (CS) are further developed. In [13], a high resolution dictionary is constructed and basis pursuit algorithm is used to find the coefficient of each atom. However, this method is limited because the dictionary construction method needs HRMS training images, which may not be available. To deal with this problem, a practical fusion method is proposed in [14]. Besides, Zhu presented the SparseFI algorithm [15] from the view of image super resolution [16]. SparseFI cannot require the HRMS images, and it explores the sparse representation of MS image patches in a dictionary trained only from the Pan image. Meanwhile, SparseFI algorithm does not assume any spectral composition model of the Pan image and gives robust performance against spectral model errors. In order to exploit the local similarity between the MS and PAN images, Jiang et al. [17] proposed the two steps sparse coding method.

However, these methods cannot take into account the local geometrical similarity within the images, which has already been used in many image processing and computer vision applications. In paper [18], a local geometrical similarity is considered as a prior in the low-resolution image to find a more accurate high-resolution image. They proposed to use the piecewise autoregressive models to characterize the local image structures.

In our work, the structure similarity prior within a local region in the Pan image is employed to regularize the solution space to obtain a more accurate solution. Then, the prior is embedded into the LRMS to enhance the spatial resolution. In order to capture better geometrical structure information, the steerable kernel [19], which can capture the more useful information about local similarity compared with the Gaussian kernel, is used to calculate the similarity coefficients in a local window. Finally, the desired HRMS is efficiently obtained via the gradient descent method.

Compared with available pansharpening technologies, the contribution of our work is twofold: 1) PLGS can 
capture better geometrical structure information, such as orientation information and geometric textures. 2) In order to obtain a more accurate solution, the structure similarity prior within a local region in the Pan image is employed to regularize the solution space. 3) The prior is embedded into the LRMS image to enhance the spatial resolution. Consequently the obtained HRMS images have fewer distortions in both spatial and spectral domains. Some experiments are taken on several datasets collected from QuickBird and IKONOS, and the experimental results show that the proposed PLGS method is effective and outperforms its counterparts.

The remainder of the paper is organized as follows. Section 2 describes the geometric structure information exploration via steerable kernel. Section 3 describes the fusion model in details and the optimization algorithm is also given. The experimental results are provided in Section 4. In Section 5, the conclusion is given.

\section{GEOMETRIC STRUCTURE INFORMATION EXPLORATION VIA STEERABLE KERNEL}

In this section we discuss the construction of steerable kernel. In order to reduce the color distortion and enhance the spatial information of fused images, we propose a Pansharpening method via Local Geometrical Similarity (PLGS). First, the structure similarity prior within a local region in the Pan image is employed to regularize the solution space to obtain a more accurate solution. Then, the prior is embedded into the LRMS image to enhance the spatial resolution. In our method, we first define the steerable kernel of the pixel $i$ as [19],

$$
\begin{aligned}
& K_{H_{i}^{\text {ster }}}\left(x_{i}-x\right)= \\
& =\frac{\sqrt{\operatorname{det}\left(\boldsymbol{C}_{i}\right)}}{2 \pi h^{2} \mu_{i}^{2}} \exp \left(-\frac{\left(x_{i}-x\right)^{\mathrm{T}} C_{i}\left(x_{i}-x\right)}{2 h^{2} \mu_{i}^{2}}\right)
\end{aligned}
$$

where $x_{i} \in Z^{2}$ is the coordinate vector of the pixel $i$, $x \in Z^{2}$ represents the coordinates of pixels in the search window $S^{i}, h$ is the global smoothing parameter, $\mu_{i}$ which is a scalar, captures the local density of data. $\boldsymbol{C}_{i}$ is a gradient covariance matrix, and $\boldsymbol{C}_{i}$ is related with the local edge structure at the pixel $i$. The estimation of $\boldsymbol{C}_{i}$ can be written as:

$$
\boldsymbol{C}_{i} \approx\left[\begin{array}{ll}
\sum_{x_{j} \in \omega_{i}} f_{u}\left(x_{j}\right) f_{u}\left(x_{j}\right) & \sum_{x_{j} \in \omega_{i}} f_{u}\left(x_{j}\right) f_{v}\left(x_{j}\right) \\
\sum_{x_{j} \in \omega_{i}} f_{v}\left(x_{j}\right) f_{u}\left(x_{j}\right) & \sum_{x_{j} \in \omega_{i}} f_{v}\left(x_{j}\right) f_{v}\left(x_{j}\right)
\end{array}\right]
$$

where, $\omega_{i}$ is a local window around the pixel $i, f_{u}(\cdot)$ and $f_{v}(\cdot)$ are the gradients along directions $u$ and $v, \boldsymbol{C}_{i}$ is decomposed into $\boldsymbol{C}_{i}=\gamma_{i} \boldsymbol{U}_{\theta_{i}} \wedge \boldsymbol{U}_{\theta_{i}}^{\mathrm{T}}$. Where, $\boldsymbol{U}_{\theta_{i}}$ is a rotation matrix and $\wedge_{i}$ is an elongation matrix. $\boldsymbol{U}_{\theta_{i}}$ and $\wedge_{i}$ can be written as

$\boldsymbol{U}_{\theta_{i}}=\left[\begin{array}{cc}\cos \theta_{i} & \sin \theta_{i} \\ -\sin \theta_{i} & \cos \theta_{i}\end{array}\right], \quad \wedge_{i}=\left[\begin{array}{cc}\sigma_{i} & 0 \\ 0 & \sigma_{i}^{-1}\end{array}\right]$,

$\gamma_{\mathrm{i}}, \theta_{\mathrm{i}}, \quad \sigma_{\mathrm{i}}$ are the scaling, rotation, and elongation parameters respectively.

Steerable kernel can be considered as evolving from Gaussian kernels, and the evolutionary process is shown in Fig. 1. First, the circular kernel is elongated by the elongation matrix $\wedge_{i}$, and its length along the major and minor axes is determined by $\sigma_{\mathrm{i}}$. Second, the elongated kernel is rotated by the matrix $\boldsymbol{U}_{\theta_{i}}$. Finally, the kernel is scaled by the scaling parameter $\gamma_{\mathrm{i}}$.
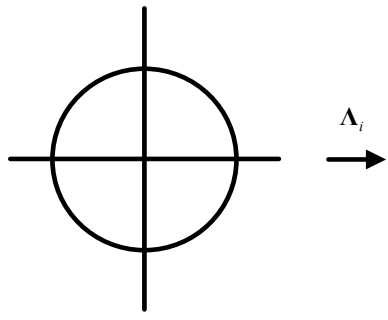

3

FUSION MODEL

As we all know, LRMS and Pan images are simultaneously produced from the same satellites. According to the observation model of satellites, the LRMS can be regarded as the result that is spatially degraded from the HRMS image. So, the relationship between LRMS and HRMS images is formulated as the

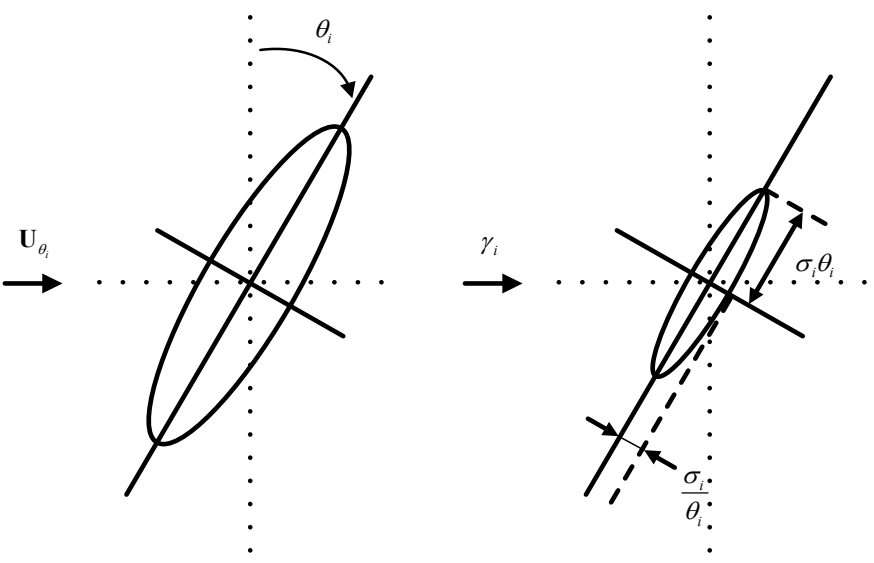

following equation:

$\boldsymbol{X}=\boldsymbol{D} \boldsymbol{Z}+\boldsymbol{v}_{1}$

where $\boldsymbol{X}$ and $\boldsymbol{Z}$ are the LRMS and HRMS images, respectively. $\boldsymbol{D}$ is the spatial degradation matrix, $\boldsymbol{v}_{1}$ is the noise. It can be noted that each channel of the LRMS and HRMS images is arranged in lexicographic order and 
placed into $\boldsymbol{X}$ and $\boldsymbol{Z}$.

The Pan image can be regarded as the result of spectral degradation from the HRMS images according to the same observation model, since the spectral responses of the LRMS images are overlapped with that of Pan image. Therefore, the degradation model between HRMS and Pan images can be written as:

$\boldsymbol{Y}=\boldsymbol{Z S}+\boldsymbol{v}_{2}$

where $\boldsymbol{Y}$ is the Pan image and $\boldsymbol{v}_{2}$ is the noise, $\boldsymbol{S}$ is the spectral degradation matrix. Then, the HRMS images can be obtained by (3) and (4) and the objective function can be written as:

$$
\hat{\boldsymbol{Z}}=\arg \min _{\boldsymbol{Z}}\left\{\frac{1}{2}\|\boldsymbol{X}-\boldsymbol{D} \boldsymbol{Z}\|_{2}^{2}+\frac{\alpha}{2}\|\boldsymbol{Y}-\boldsymbol{Z} \boldsymbol{S}\|_{2}^{2}\right\}
$$

In order to obtain a more accurate solution, the geometrical similarity prior in the Pan image is used. It is known to us that the target pixel can be linearly represented by adjacent pixels and the prior is widely used on many applications, such as image denoising [20]. Although the structure information in the Pan image may be different than those in the LRMS images, they cover the same area. There is similar structure information between Pan and LRMS images. Thus, the geometrical structure in Pan image can be used to regularize the solution to (5).

In our proposed method, the steerable kernel is used to calculate the weights, which can efficiently capture the spatial structure and the edge information of the Pan image. Thus, through the steerable kernel of pixel $i$, the representation weights of its neighbors in a local window can be defined as:

$w_{i j}=\frac{1}{l(i)} \exp \left(-\frac{\left\|y\left(N_{i}\right)-y\left(N_{j}\right)\right\|_{K}^{2}}{h^{2}}\right) K(j)$

where

$l(i)=\sum_{j \in S_{i}}\left(-\frac{\left\|y\left(N_{i}\right)-y\left(N_{j}\right)\right\|_{K}^{2}}{h^{2}}\right) \exp (-K(j))$

is the coordinate of pixel $i$ and $S_{i}$ is the set of coordinates of pixels in the local window, $h$ is the global smoothing parameter, $\|\cdot\|_{K}^{2}$ is the Euclidean distance weighted by the steerable kernel $K$. The steerable kernel is written as:

$$
K(j)=\frac{\sqrt{\operatorname{det}\left(\boldsymbol{C}_{i}\right)}}{2 \pi h^{2}} \exp \left(-\frac{\left(x_{i}-x_{j}\right)^{\mathrm{T}} \boldsymbol{C}_{i}\left(x_{i}-x_{j}\right)}{2 h^{2}}\right)
$$

where $\boldsymbol{C}_{i}$ is a gradient covariance matrix computed by the differences in the local window, which can capture the local edge structure at the pixel $i ; x_{i}$ and $x_{j}$ denote the coordinates of pixels $i$ and $j$; $\mathrm{T}$ is the transpose operator.
Through the weights from the steerable kernel, the image can be represented by itself according to the local similarity prior. Therefore, the local similarity can be written as a regularization term:

$$
\hat{\boldsymbol{Z}}=\arg \min _{\boldsymbol{Z}}\|\boldsymbol{Z}-\boldsymbol{A} \boldsymbol{Z}\|_{2}^{2}
$$

where $\boldsymbol{A}$ is the coefficient matrix which is formed by the similarity weights calculated by (6). We can combine (5) and $(8)$ to estimate the desired HRMS image $(\boldsymbol{Z})$. Thus, the objective function can be formulated as:

$\hat{\boldsymbol{Z}}=\arg \min _{\boldsymbol{Z}}\left\{\frac{1}{2}\|\boldsymbol{X}-\boldsymbol{D} \boldsymbol{Z}\|_{2}^{2}+\frac{\alpha}{2}\|\boldsymbol{Y}-\boldsymbol{Z} \boldsymbol{S}\|_{2}^{2}+\frac{\beta}{2}\|\boldsymbol{Z}-\boldsymbol{A} \boldsymbol{Z}\|_{2}^{2}\right\}$

where $\alpha$ and $\beta$ are the balance parameters.

Obviously, Eq. (9) can be optimized by the gradient descent method. The update rule can be written as:

$$
\begin{aligned}
& \boldsymbol{Z}^{t+1}=\boldsymbol{Z}^{t}+\lambda\left(\boldsymbol{D}^{\mathrm{T}} \boldsymbol{X}+\alpha \boldsymbol{Y} \boldsymbol{S}^{\mathrm{T}}-\alpha \boldsymbol{Z} \boldsymbol{S}^{\mathrm{T}} \boldsymbol{S}-\right. \\
& \left.-\left(\boldsymbol{D}^{\mathrm{T}} \boldsymbol{D}-\beta(\boldsymbol{I}-\boldsymbol{A})^{\mathrm{T}}(\boldsymbol{I}-\boldsymbol{A})\right) \boldsymbol{Z}\right)
\end{aligned}
$$

where $t$ is iteration times. $\lambda$ is the step size for gradient descent, and I is the identity matrix. In the first step, Z is initialized to the bicubic interpolation version of the LRMS images. The iterations are terminated until the maximum number is satisfied or the value of relative error is reached.

\section{SIMULATIONS AND ANALYSIS}

In this section, we analyze the fusion model on datasets from different satellites. First, the proposed method is compared with some classic methods, such as GIHS [4], PCA [5], DWT [9] and AWLP [12] on different satellite datasets and the fusion results are shown in Figs. 2-5. To verify the performance of our proposed algorithm in both spectral and spatial features, some experiments are compared by visual quality subjectively and by some guidelines, such as Spectral Angle Mapper (SAM) [21], Erreur Relative Globale Adimensionnelle de Synthèse (ERGAS) [22], $\mathrm{Q}_{4}$ [23] and Universal Image Quality Index (UIQI) [24]. For the first two metrics, SAM and $E R G A S$ will be close to 0 if the fusion result is better. For the other two metrics, the values closer to 1 imply better results and the best values of them are 1 .

Besides, the parameter about steerable kernel $h$ is set as 3 . The size of the search window is $9 \times 9$. The parameters about the optimization algorithm $\alpha$ and $\beta$ are set as 0.4 and 0.02 , respectively. The step size $\lambda$ is 1 and the maximum number of iterations (Iter) is 20. The relative error is empirically set as $10^{-6}$ for an accurate solution. Moreover, the parameters in the compared methods are obtained from the literature.

\subsection{Experiments on QuickBird Datasets}

In this subsection, the performance of the proposed methods is evaluated on QuickBird images as shown in Figs. 2-3. The QuickBird images are the scenes of Xi'an, 
China and Sundarbans, India. The spatial resolution of LRMS and Pan images from QuickBird satellite are $9.6 \mathrm{~m}$ and $2.4 \mathrm{~m}$. The first two images in Figs. 2 and 3 are the LRMS and Pan images which will be fused. The third image among them is the reference image which is used to evaluate the performance of each method. The fused results of various methods are shown in Figs. 2-3(d)-(h). The fusion results will be better if the result is closer to the reference image.

From Fig. 2, we can see that significant spectral distortions are produced by GIHS and PCA, but their spatial details are better than DWT and AWLP, which can be found in some local urban areas. DWT and AWLP have a better performance in spectral preservation. For example, the color of the tree in AWLP is more natural than those of GIHS and PCA in Fig. 2, but the ringing effects can be found in their results which can be observed in the vegetable areas. However, our proposed method can preserve the spatial and spectral information more efficiently. In Fig.3, the proposed method has the best spectral quality. AWLP also provides a good spectral performance, but, AWLP and DWT suffer from ringing and aliasing artifacts on the vegetable areas in the fused images.

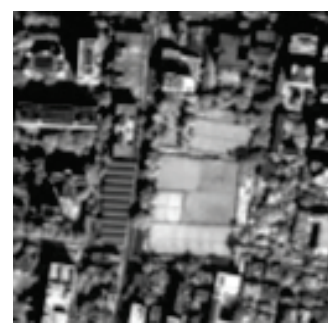

(a)

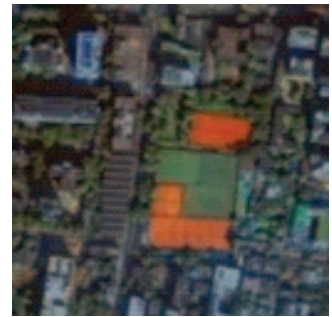

(e)

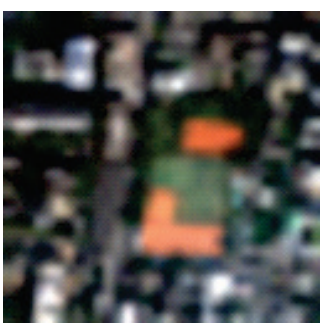

(b)

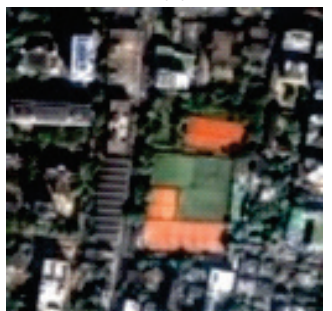

(f)

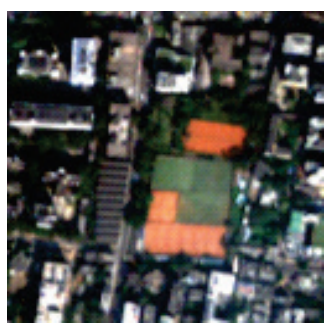

(c)

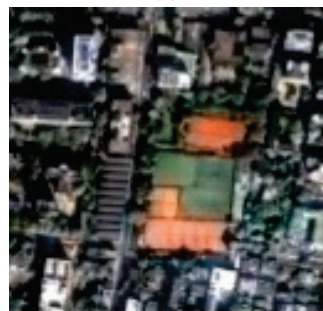

(g)

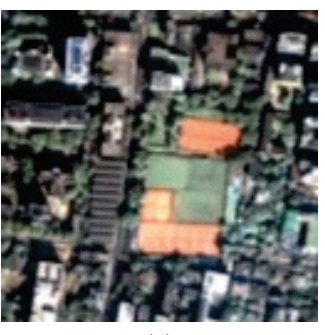

(d)

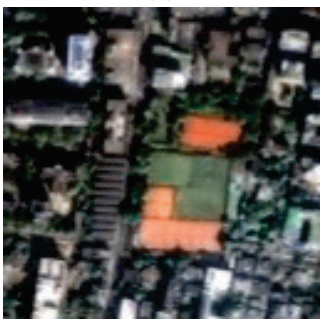

(h)

Figure 2 Fused results by different methods. (a) Pan. (b) LRMS. (c) Reference image. (d) GIHS. (e) PCA. (f) AWLP. (g) DWT. (h) PLGS

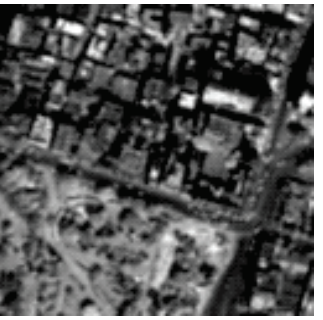

(a)

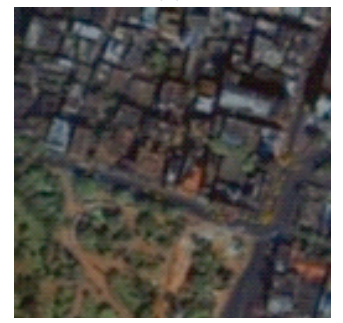

(e)

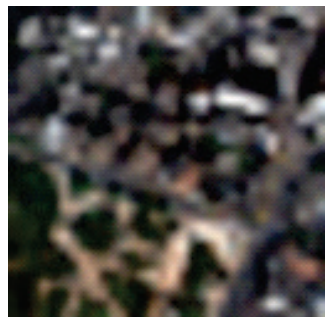

(b)

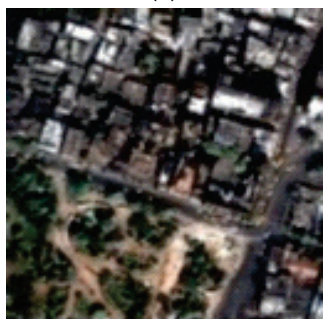

(f)

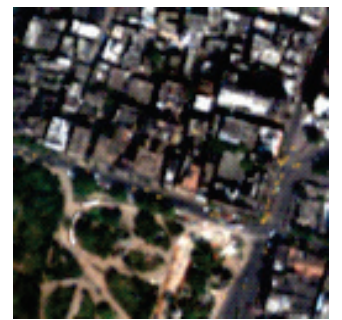

(c)

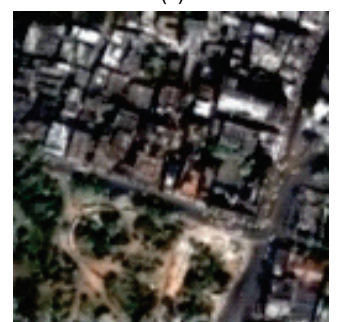

(g)

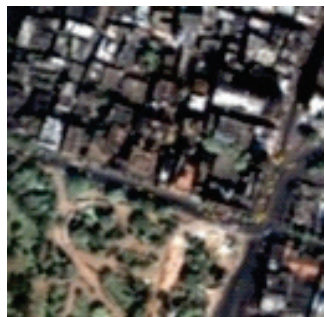

(d)

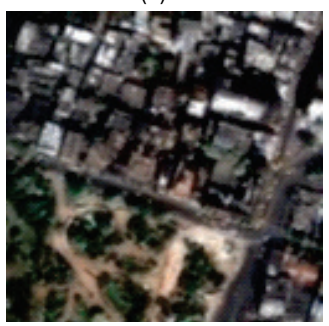

(h)

Figure 3 Fused results by different methods. (a) Pan. (b) LRMS. (c) Reference image. (d) GIHS. (e) PCA. (f) AWLP. (g) DWT. (h) PLGS.

Table 1 The quantitative results of the images in Fig. 2

\begin{tabular}{|c|c|c|c|c|c|c|}
\hline Metric & Band & GIHS & PCA & AWLP & DWT & PLGS \\
\hline \multirow{4}{*}{ UIQI } & $\mathrm{R}$ & 0.8659 & 0.6468 & 0.9196 & 0.8820 & $\mathbf{0 . 9 1 9 7}$ \\
\cline { 2 - 7 } & $\mathrm{G}$ & 0.8404 & 0.6131 & $\mathbf{0 . 9 1 1 0}$ & 0.8784 & 0.9108 \\
\cline { 2 - 7 } & $\mathrm{B}$ & 0.7920 & 0.6293 & 0.8709 & 0.8268 & $\mathbf{0 . 8 8 1 2}$ \\
\cline { 2 - 7 } & NIR & 0.9335 & 0.8242 & 0.9624 & 0.9490 & $\mathbf{0 . 9 6 2 5}$ \\
\cline { 2 - 7 } & Avg. & 0.8580 & 0.6783 & 0.9160 & 0.8840 & $\mathbf{0 . 9 1 8 6}$ \\
\hline \multicolumn{2}{|c|}{$\mathrm{Q}_{4}$} & 0.7446 & 0.5393 & $\mathbf{0 . 7 8 2 1}$ & 0.7638 & 0.7801 \\
\hline \multicolumn{2}{|c|}{ SAM } & 11.9840 & 11.8341 & 15.0592 & 17.2199 & $\mathbf{1 1 . 2 4 1 5}$ \\
\hline \multicolumn{2}{|c|}{ ERGAS } & 10.5102 & 11.6300 & 7.5709 & 8.8245 & $\mathbf{7 . 4 7 8 9}$ \\
\hline
\end{tabular}

Table 2 The quantitative results of the images in Fig. 3

\begin{tabular}{|c|c|c|c|c|c|c|}
\hline Metric & Band & GIHS & PCA & AWLP & DWT & PLGS \\
\hline \multirow{4}{*}{ QI } & R & 0.8742 & 0.5725 & 0.9177 & 0.8869 & $\mathbf{0 . 9 2 1 4}$ \\
\cline { 2 - 7 } & G & 0.8686 & 0.5651 & 0.9202 & 0.8969 & $\mathbf{0 . 9 2 5 6}$ \\
\cline { 2 - 7 } & B & 0.8293 & 0.5594 & 0.8862 & 0.8532 & $\mathbf{0 . 8 9 1 2}$ \\
\cline { 2 - 7 } & NIR & 0.9196 & 0.8127 & 0.9441 & 0.9269 & $\mathbf{0 . 9 5 0 3}$ \\
\cline { 2 - 7 } & Avg. & 0.8730 & 0.6274 & 0.9170 & 0.8910 & $\mathbf{0 . 9 2 2 1}$ \\
\hline \multicolumn{2}{|c|}{ Q $_{4}$} & 0.8126 & 0.5436 & $\mathbf{0 . 8 4 6 3}$ & 0.8304 & 0.8394 \\
\hline \multicolumn{2}{|c|}{ SAM } & 10.6949 & 11.0640 & 13.0783 & 14.9749 & $\mathbf{1 0 . 4 1 2 8}$ \\
\hline \multicolumn{2}{|c|}{ ERGAS } & 9.1744 & 11.8159 & 7.1486 & 8.1523 & $\mathbf{7 . 0 4 5 6}$ \\
\hline
\end{tabular}


Besides, the quantitative values are given in Tabs. 1-2. It is obvious that our proposed method produces the best values in terms of UIQI, SAM and ERGAS in Tab. 1. Although the best value of $\mathrm{Q}_{4}$ is given by AWLP in Tab.1, our proposed method has better performance generally. In conclusion, the whole performance of our proposed method is better than the other methods. We can find that the quantitative result in Tab. 2 is consistent with those of Tab. 1. Our proposed method gives best values for most metrics.

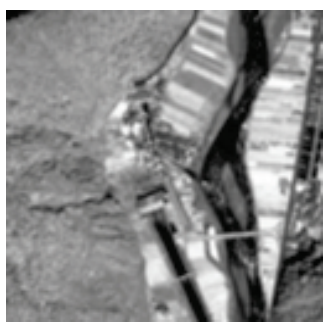

(a)

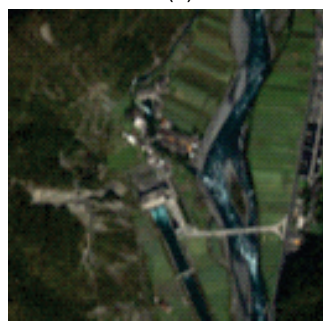

(c)

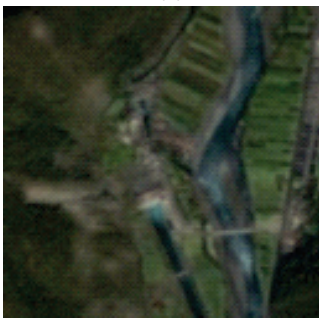

(e)

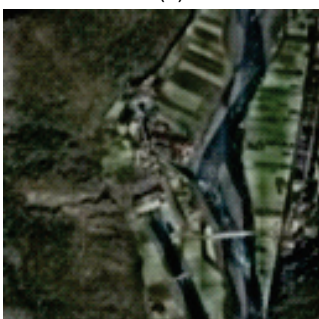

(g)

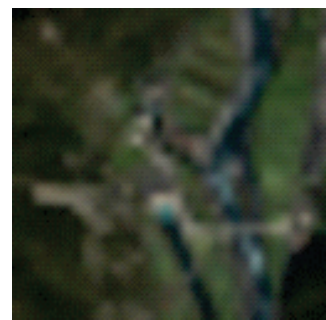

(b)

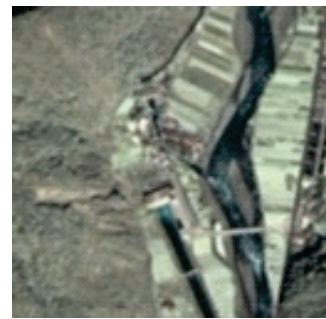

(d)

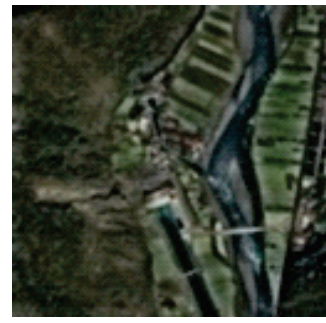

(f)

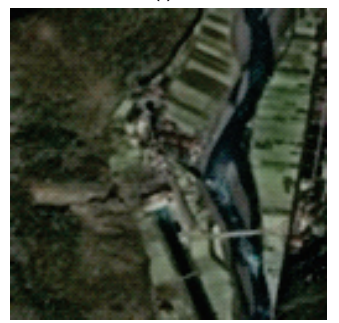

(h)
Figure 4 Fused results by different methods. (a) Pan. (b) LRMS. (c) Reference image. (d) GIHS. (e) PCA. (f) AWLP. (g) DWT. (h) PLGS.

\subsection{Experiments on IKONOS Datasets}

In this subsection, the performance of the proposed methods is evaluated on IKONOS images. The datasets from IKONOS satellite are from Sichuan, China and tested on different methods whose results are displayed in Figs. 4 and 5. The fused results of various methods are shown in Figs. 4-5(d)-(h). In the same way, the LRMS and Pan images are produced from HRMS and high resolution Pan images and the HRMS image is regarded as a reference image. The LRMS image with resolution $16 \mathrm{~m}$ and the Pan image with resolution of $4 \mathrm{~m}$ are provided by IKONOS satellite.

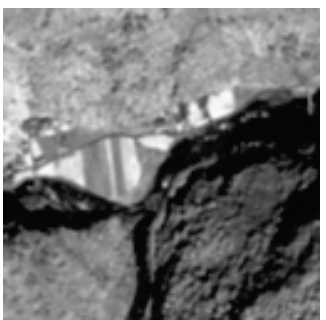

(a)

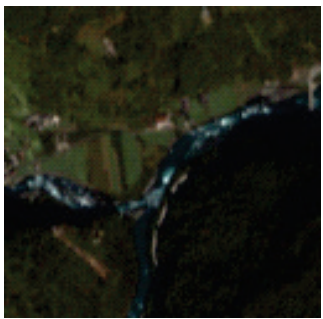

(c)

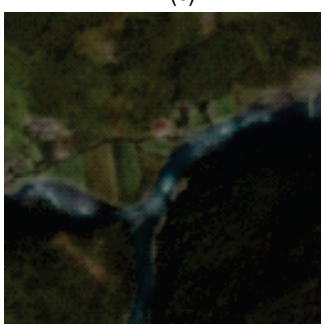

(e)

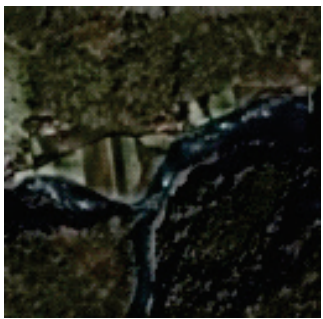

(g)

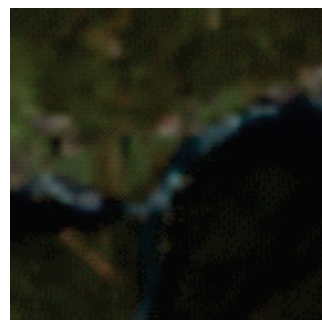

(b)

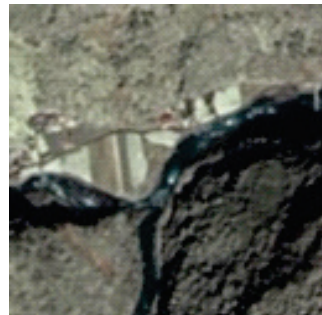

(d)

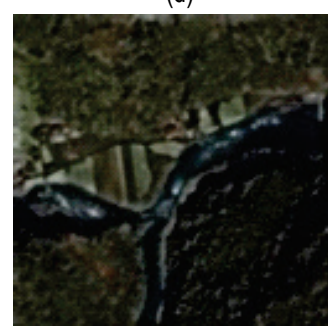

(f)

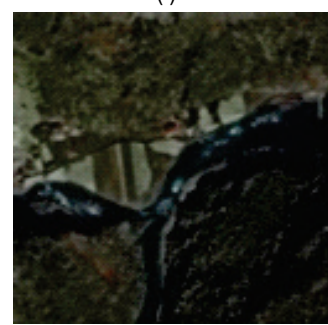

(h)
Figure 5 Fused results by different methods. (a) Pan. (b) LRMS. (c) Reference image. (d) GIHS. (e) PCA. (f) AWLP. (g) DWT. (h) PLGS.

Table 3 The quantitative results of the images in Figure 4

\begin{tabular}{|c|c|c|c|c|c|c|}
\hline Metric & Band & GIHS & PCA & AWLP & DWT & PLGS \\
\hline \multirow{4}{*}{ UIQI } & R & 0.3043 & 0.3784 & 0.5180 & 0.4459 & $\mathbf{0 . 5 1 8 7}$ \\
\cline { 2 - 7 } & G & 0.3364 & 0.4012 & 0.5487 & 0.4924 & $\mathbf{0 . 5 5 4 8}$ \\
\cline { 2 - 7 } & $\mathrm{B}$ & 0.1608 & 0.4345 & 0.4347 & 0.3606 & $\mathbf{0 . 4 4 1 2}$ \\
\cline { 2 - 7 } & NIR & 0.8215 & 0.8412 & $\mathbf{0 . 8 8 6 8}$ & 0.8681 & 0.8803 \\
\cline { 2 - 7 } & Avg. & 0.4057 & 0.5138 & 0.5970 & 0.5417 & $\mathbf{0 . 5 9 8 7}$ \\
\hline \multicolumn{2}{|c|}{$\mathrm{Q}_{4}$} & 0.5376 & 0.5443 & 0.5666 & 0.5361 & $\mathbf{0 . 5 6 9 4}$ \\
\hline \multicolumn{2}{|c|}{ SAM } & 12.3001 & 10.6421 & $\mathbf{1 0 . 1 9 3 1}$ & 12.0809 & 12.6019 \\
\hline \multicolumn{2}{|c|}{ ERGAS } & 24.0075 & 13.5391 & $\mathbf{9 . 6 9 8 9}$ & 10.6311 & 10.9789 \\
\hline
\end{tabular}


Compared with the reference image, we can see that some distortions in spectral information are produced in the results of GIHS and PCA in Fig. 4. In addition, DWT and AWLP can provide good color information. Our method produces clearer spatial details, and the spectral information is more consistent with that of the HRMS image. From the fused images shown in Fig. 5, it can be observed that the spectral information of the results of AWLP and our proposed method is close to the reference image. Some spatial details are lost in DWT and GIHS, and blurring effects can be found. Moreover, PCA is poor contrast. Compared with the reference image, the fused images of our proposed method have better spatial structures and spectral information.

Table 4 The quantitative results of the images in Fig. 5

\begin{tabular}{|c|c|c|c|c|c|c|}
\hline Metric & Band & GIHS & PCA & AWLP & DWT & PLGS \\
\hline \multirow{4}{*}{ UIQI } & R & 0.4003 & 0.5574 & $\mathbf{0 . 6 1 0 3}$ & 0.5772 & 0.6093 \\
\cline { 2 - 7 } & G & 0.4007 & 0.5566 & $\mathbf{0 . 6 1 5 4}$ & 0.5868 & 0.6148 \\
\cline { 2 - 7 } & B & 0.2388 & $\mathbf{0 . 5 5 6 3}$ & 0.4309 & 0.3960 & 0.4312 \\
\cline { 2 - 7 } & NIR & 0.8488 & 0.6807 & $\mathbf{0 . 9 1 8 1}$ & 0.9080 & 0.9003 \\
\cline { 2 - 7 } & Avg. & 0.4721 & 0.5877 & $\mathbf{0 . 6 4 3 6}$ & 0.6170 & 0.6389 \\
\hline \multicolumn{2}{|c|}{ Q $_{4}$} & 0.5532 & 0.5343 & 0.5947 & 0.5738 & $\mathbf{0 . 6 0 1 4}$ \\
\hline \multicolumn{2}{|c|}{ SAM } & 15.9698 & 16.1192 & 16.6207 & 17.9946 & $\mathbf{1 5 . 6 0 1 9}$ \\
\hline \multicolumn{2}{|c|}{ ERGAS } & 35.1094 & 19.5391 & 13.4825 & 14.3674 & $\mathbf{1 2 . 9 7 8 9}$ \\
\hline
\end{tabular}

Tabs. 3 and 4 report the values of UIQI, $Q_{4}$, SAM and ERGAS. For these metrics in Tab. 3, AWLP produces the best value in SAM and ERGAS. However, our proposed method provides the best UIQI and $\mathrm{Q}_{4}$, which implies that the fused image of our proposed method has better performance in spectral preservation. As we can see from Tab. 4, our method provides the best results in terms of $\mathrm{Q}_{4}, \mathrm{SAM}$ and ERGAS. The best values of UIQI are from AWLP. However, combining the visual results and the metric values, we can see that our PLGS method produces better fused images.

\subsection{Analysis of Parameters}

In this section, we analyze the influences of parameters $\alpha$ and $\beta$ on the fusion results of the proposed method. The test images are showed in Sections 4.1 and 4.2. In Fig. 6, we plot the metrics, UIQI, $\mathrm{Q}_{4}, \mathrm{SAM}$ and ERGAS against the parameter with one parameter fixed while another varies. Fig. 6(a) shows the variation of $\alpha$ and with fixed $\beta$. The result of $\beta$ is displayed in Fig. 6(b). In this test, $\alpha$ varies from 0.1 to 1 with the step of 0.1 . $\beta$ varies from 0.01 to 0.1 with the step of 0.01 . The values of SAM and ERGAS are normalized to the range $[0,1]$ for direct comparison.

From Fig. 6, it can be seen that the fusion results vary with the variations of $\alpha$ with $\beta$. In Fig. 6(a), the best metrics, such as UIQI, $\mathrm{Q}_{4}$ and SAM, are achieved when $\alpha$ is 0.4 with the fixed $\beta$. Though ERGAS is not the best value, almost metrics behave well. In Fig. 6(b), it is obvious that the best results are given when $\beta$ is 0.06 . Thus, in our work, $\alpha$ and $\beta$ are set as 0.4 and 0.06 , respectively.

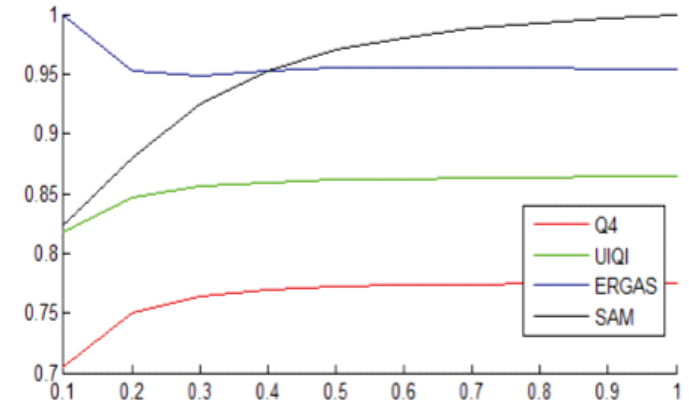

(a) the influences of $\alpha$

Figure 6 The metrics versus parameters

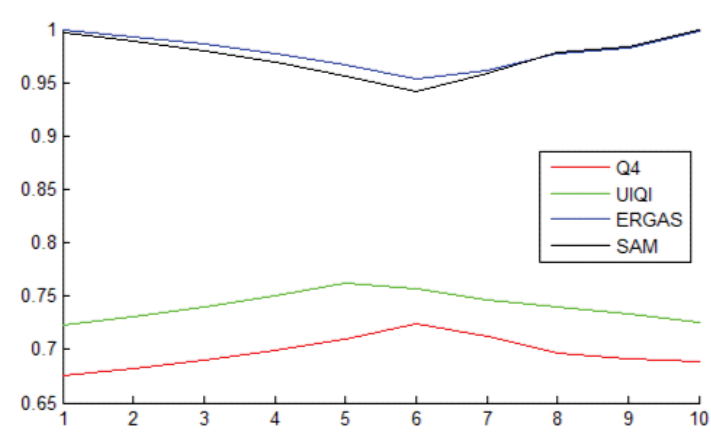

(b) the influences of $\beta$

\section{CONCLUSION}

In this paper, a novel PLGS method based on the steerable kernel is proposed to exploit the self-similarity in the image. The relationships among remote sensing images are denoted by the observation models, and PLGS method can obtain more structure information, which is extracted via the steerable kernel. The proposed method is tested on QuickBird and IKONOS datasets, and the results are compared with some popular and related pan-sharpening methods, including GIHS, PCA, AWLP, and DWT. Experimental results demonstrate that PLGS method takes longer experimental time. However, the proposed method can reduce the color distortion and preserve spatial information, and outperform some related fusion approaches in visual results and numerical guidelines.

\section{Acknowledgments}

This work was supported in part by the National Natural Science Foundation of China (no. 81473559), the Science Basic Research Program in Shaanxi Province of China (no. 16JK1823), the Natural Science Basic Research Plan in Shaanxi Province of China (no. 2017JM6086), the Science Basic Research Program in XianYang Normal University (no. 15XSYK045), the Education Scientific Program of $13^{\text {th }}$ Five-year Plan in Shaanxi Province of China (no. SGH17H196), the Teaching Reform Program in Xianyang Normal University of China (no. 2017Z014).

\section{REFERENCES}

[1] Zhong, Z. \& Pi, D. (2015). Forecasting Satellite Attitude Volatility Using Support Vector Regression with Particle 
Swarm Optimization. IAENG International Journal of Computer Science, 41(3), 153-162.

[2] Mohammadzadeh, A., Tavakoli, A., \& Zoej, M. J. V. (2010). Road Extraction Based on Fuzzy Logic and Mathematical Morphology from Pansharpened IKONOS Images. Photogrammetric Record, 21(113), 44-60. https://doi.org/10.1111/j.1477-9730.2006.00353.x

[3] Barrho, J., Hauger, J., \& Kiencke, U. (2008). Information Fusion Based on Bayesian Networks for Hazard Analysis in Machine Tool Environments. IAENG International Journal of Computer Science, 35(1), 1-9.

[4] Tu, T. M., Huang, P. S., Hung, C. L., \& Chang, C. P. (2004). A Fast Intensity-Hue-Saturation Fusion Technique with Spectral Adjustment for IKONOS Imagery. IEEE Geoscience and Remote Sensing Letters, 1(4), 309-312. https://doi.org/10.1109/LGRS.2004.834804

[5] Chavez, P. S., Sides, S. C., \& Anderson, J. A. (1991). Comparison of Three Different Methods to Merge Multiresolution and Multispectral Data: Landsat TM and SPOT Panchromatic. Photogrammetric Engineering \& Remote Sensing, 57(3), 295-303.

[6] Elkhadir, Z., Chougdali, K., \& Benattou, M. (2016). Intrusion Detection System Using PCA and Kernel PCA Methods. IAENG International Journal of Computer Science, 43(1), 72-79. https://doi.org/10.1007/978-3-319-30298-0_50

[7] Priorov, A., Tumanov, K., Volokhov, V., Sergeev, E., \& Mochalov, I. (2013). Applications of Image Filtration Based on Principal Component Analysis and Nonlocal Image Processing. IAENG International Journal of Computer Science, 40(2), 62-80.

[8] Tu, T. M., Su, S. C., Shyu, H. C., \& Huang, P. S. (2001). A New Look at IHS-Like Image Fusion Methods. Information Fusion, 2(3), 177-186. https://doi.org/10.1016/S1566-2535(01)00036-7

[9] Mallat, S. G. (1989). A Theory for Multiresolution Signal Decomposition: The Wavelet Representation. IEEE Computer Society. https://doi.org/10.1109/34.192463

[10] Burt, P. J. \& Adelson, E. H. (1987). The Laplacian Pyramid as a Compact Image Code. IEEE Transactions on Communications, 31(4), 671-679. https://doi.org/10.1016/B978-0-08-051581-6.50065-9

[11] Do, M. N. \& Vetterli, M. (2005). The Contourlet Transform: An Efficient Directional Multiresolution Image Representation. IEEE Transactions on Image Processing, 14(12), 2091-2106. https://doi.org/10.1109/TIP.2005.859376

[12] Otazu, X., González-Audícana, M., Fors, O., \& Núñez, J. (2005). Introduction of Sensor Spectral Response into Image Fusion Methods: Application to Wavelet-Based Methods. IEEE Transaction Geoscience \& Remote Sensing, 43(10), 2376-2385. https://doi.org/10.1109/TGRS.2005.856106

[13] Li, S. \& Yang, B. (2011). A New Pan-Sharpening Method Using a Compressed Sensing Technique. IEEE Transaction Geoscience \& Remote Sensing, 49(2), 738-746. https://doi.org/10.1109/TGRS.2010.2067219

[14] Jiang, C., Zhang, H., Shen, H., \& Zhang, L. (2012). A Practical Compressed Sensing Based Pan-Sharpening Method. IEEE Transaction Geoscience \& Remote Sensing Letters, 9(4), 629-633. https://doi.org/10.1109/LGRS.2011.2177063

[15] Zhu, X. X. \& Bamler, R. (2013). A Sparse Image Fusion Algorithm with Application to Pan-Sharpening. IEEE Transaction Geoscience \& Remote Sensing, 51(5), 2827-2836. https://doi.org/10.1109/TGRS.2012.2213604

[16] Pan, Z., Yu, J., Huang, H., Hu, S., Zhang, A., Ma, H., \& Sun, W. (2013). Super-Resolution Based on Compressive Sensing and Structural Self-Similarity for Remote Sensing Images. IEEE Transaction Geoscience \& Remote Sensing, 51(9), 4864-4876.
https://doi.org/10.1109/TGRS.2012.2230270

[17] Jiang, C., Zhang, H., Shen, H., \& Zhang, L. (2013). Two-Step Sparse Coding for the Pan-Sharpening of Remote Sensing Images. IEEE Selected Topics in Applied Earth Observations and Remote Sensing, 7(5), 1792-1805. https://doi.org/10.1109/JSTARS.2013.2283236

[18] Dong, W., Zhang, L., Lukac, R., \& Shi, G. (2013). Sparse Representation Based Image Interpolation with Nonlocal Autoregressive Modeling. IEEE Transaction on Image Processing, 22(4), 1382-1394. https://doi.org/10.1109/TIP.2012.2231086

[19] Takeda, H., Farsiu, S., \& Milanfar, P. (2007). Kernel Regression for Image Processing and Reconstruction. IEEE Transaction on Image Processing, 16(2), 349-366. https://doi.org/10.1109/TIP.2006.888330

[20] Dabov, K., Foi, A., Katkovnik, V., \& Egiazarian, K. O. (2007). Image Denoising by Sparse 3D Transform Domain Collaborative Filtering. IEEE Transaction on Image Processing, 16(8), 2080-2095. https://doi.org/10.1109/TIP.2007.901238

[21] Alparone, L., Wald, L., Chanussot, J., Thomas, C., Gamba, P., \& Bruce, L. M. (2007). Comparison of Pansharpening Algorithms: Outcome of the 2006 GRS-S Data-Fusion Contest. IEEE Transaction Geoscience \& Remote Sensing, 45(10), 3012-3021. https://doi.org/10.1109/TGRS.2007.904923

[22] Khan, M. M., Alparone, L., \& Chanussot, J. (2009). Pansharpening Quality Assessment Using the Modulation Transfer Functions of Instruments. IEEE Transaction Geoscience \& Remote Sensing, 47(11), 3880-3891. https://doi.org/10.1109/TGRS.2009.2029094

[23] Alparone, L., Baronti, S., Garzelli, A., \& Nencini, F. (2004). A Global Quality Measurement of Pan-Sharpened Multispectral Imagery. IEEE Transaction Geoscience \& Remote Sensing Letters, 1(4), 313-317. https://doi.org/10.1109/LGRS.2004.836784

[24] Wang, Z. \& Bovik, A. C. (2002). A Universal Image Quality Index. IEEE Signal Processing Letters, 9(3), 81-84. https://doi.org/10.1109/97.995823

\section{Contact information}

Hong LI, PhD

(Corresponding author)

School of Computer Science, XianYang Normal University

XianYang, Shaanxi, 712000, China

E-mail: honglishining@163.com

\section{Fenxia WU,}

School of Computer Science, XianYang Normal University

XianYang, Shaanxi, 712000, China

E-mail: 790025668@qq.com

\section{Xiaobo ZHANG, PhD}

School of Computer Science, XianYang Normal University XianYang, Shaanxi, 712000, China

E-mail: 595712278@qq.com 